\title{
Development Prospects and Enginering of Quantum Communication Systems in the Atmosphere
}

\author{
A.V.Shkalikov ${ }^{1 a}$, I.Z.Latypov ${ }^{1}$, D.O.Akatyev ${ }^{2}$, and A.A.Kalachev ${ }^{1}$ \\ ${ }^{1}$ Kazan E. K. Zavoisky Physical -Technical Institute, 10/7 Sibirsky tract Str. Kazan 420029, Russia \\ ${ }^{2}$ Kazan Federal University, 18Kremlevskay Str.Kazan 420008, Russia
}

\begin{abstract}
Scheme of quantum key distribution with polarization coding based on parametric scattering in the crystal PPKTP was desined. The possibility of application of this scheme, together with atmospheric satellites was researched. The possibility of changes to the coding scheme using the orbital angular momentum of the photon was analyzed.
\end{abstract}

Keywords: quantum cryptography, quantum key distribution, BB84, spontaneous parametric down conversion.

In recent decades, along with the usual secret transmission of information more and more attention is paid to the transfer of information on the quantum communication channels, where secrecy is due to the principles of quantum physics. Single photons are the main carriers of information in such systems, the very same information is encoded in either polarization or orbital angular momentum. Among all the possible methods of generating single-photon states unique place is a process of spontaneous parametric down conversion scattering (SPDC) light. This is due to the broad range of capabilities for managing and temporary form of prepared single-photon states.

In this paper, a scheme for transmitting secret information based on quantum key distribution protocol BB84 [1] proposed by Bennett and Brassard more than 30 years ago. As a rule, the practical application of quantum key distribution is limited to a distance of $150 \mathrm{~km}$. This is due to the large background noise semiconductor single-photon detectors. To overcome this limitation, it proposed to use the atmospheric satellites. At the heart of the developed scheme is the preparation of single photons by the SPDC in a crystal with a periodic polarization nonlinearities PPKTP. One of the generated photons is in $770 \mathrm{~nm}$ and corresponds to one of the atmospheric transmission windows. The second photon is 1.5 microns is used as a herald.

\section{References}

1. C. Bennett, G. Brassard, Proceedings of IEEE International Conference on Computers, Systems and Signal Processing (Institute of Electrical and Electronics Engineers, New York, 1984), P. 175-179

\footnotetext{
${ }^{\text {a }}$ Corresponding author: andrei_vs@rambler.ru
} 